\title{
Supportive care needs of multicultural patients with cancer in the United Arab Emirates
}

\author{
Satish Chandrasekhar Nair ${ }^{1}$, Hassan Jaafar ${ }^{2}$, Mohamed Jaloudi², Khaled Qawasmeh ${ }^{3}$, Afra AIMarar ${ }^{4}$ and Halah Ibrahim ${ }^{5}$ \\ ${ }^{1}$ Department of Academic Affairs, Tawam Hospital_Johns Hopkins Medicine Affiliate, Al Ain 15258, Abu Dhabi, United Arab Emirates \\ ${ }^{2}$ Department of Medical Oncology, Tawam Hospital_Johns Hopkins Medicine Affiliate, Al Ain 15258, Abu Dhabi, United Arab Emirates \\ ${ }^{3}$ Department of Nursing, Tawam Hospital_Johns Hopkins Medicine Affiliate, Al Ain 15258, Abu Dhabi, United Arab Emirates \\ ${ }^{4}$ Department of Surgical Oncology, Tawam Hospital-Johns Hopkins Medicine Affiliate, Al Ain 15258, Abu Dhabi, United Arab Emirates \\ 5Johns Hopkins Graduate School of Education, Baltimore, MD 21218, USA
}

Correspondence to: Satish Chandrasekhar Nair. Email: satchi2000@.outlook.com and schandra@seha.ae

\begin{abstract}
Despite the high prevalence of cancer in the Middle East, there is limited published data reporting the needs of cancer patients in this region of the world. The purpose of this study is to assess the unmet supportive care needs of oncology patients in the United Arab Emirates (UAE). From December 2014 to December 2016, a cross-sectional survey of cancer patients was conducted at a large tertiary care hospital and an oncology referral centre in the UAE, using a validated Arabic translation of the supportive care needs survey-short form (SCNS-SF34-A), assessing cancer-specific perceived needs across five domains: psychological, health system information, patient care and support, physical and daily living and sexuality. Chi-square test and Pearson's correlation coefficient were used to assess the association between variables. Participant responses were tabulated as mean \pm standard error of the mean (SEM). The response rate was $78 \%$
\end{abstract}

Published: 29/05/2018

Received: 29/01/2018

ecancer 2018, 12:838 https://doi.org/10.3332/ecancer.2018.838

Copyright: $\odot$ the authors; licensee ecancermedicalscience. This is an Open Access article distributed under the terms of the Creative Commons Attribution License (http://creativecommons.org/licenses/by/3.0), which permits unrestricted use, distribution, and reproduction in any medium, provided the original work is properly cited. 
(210/268). Five of the 10 items from the psychological domain constituted the 10 most prevalent unmet moderate or high needs, followed by physical and daily living needs $(3.04 \pm 0.029, p<0.001)$, health system information $(3.03 \pm 0.02, p<0.001)$, patient care and support $(2.95 \pm 0.24, p<0.001)$, with low sexuality needs $(1.79 \pm 0.08, p<0.001)$. Women had significantly higher psychological unmet needs. Cultural differences were noted only in the health system information domain. Improvements in mental health services, development of multidisciplinary cancer care teams, introduction of cancer support groups and fully engaging women in all treatment decisions are feasible and easy to implement interventions that can significantly improve the care and wellbeing of oncology patients in the UAE.

Keywords: cancer, supportive care needs, Middle East, psychological needs, oncology

\section{Introduction}

Cancer is the second leading cause of death in the world, with over 8 million fatalities annually [1]. Despite advances in both prevention and treatment options, the cancer burden continues to rise globally, fuelled by an ageing population and increasing lifestyle-related risk factors [2]. The prevalence of cancer in the Middle East is high and growing [3]. The World Health Organization (WHO) reports that within the next 15 years, the Middle East is likely to experience the highest increase in cancer incidence among WHO regions, with predicted increases as high as $100-180 \%[3,4]$. Recent improvements in multi-regimen modalities have resulted in significant increases in disease-free survival rates [5, 6], but do not address the supportive care needs of oncology patients. There is a large body of literature documenting that cancer patients are at risk for adverse physical, psychological, spiritual and social problems throughout the course of their diagnosis and treatment [7]. Physical complaints, including illness and disability, psychological concerns manifested as fear of pain or death and social issues, such as family dynamics, all affect cancer patients, with levels of accompanying distress that vary from patient to patient [8]. The distress can interfere substantially with comfort, quality of life and the ability to make suitable decisions or to adhere to treatment [9]. Negative patient and family experiences can also be a reflection of health care delivery systems that do not account for patient and family needs [10]. Patient needs affect all types and stages of cancer [11]. Supportive care needs also differ across countries and cultures [12]. Among ethnically diverse groups of patients, culture has been shown to influence perceptions and coping mechanisms related to disease management [13]. Therefore, cultural beliefs and values can serve as important determinants of clinical outcomes following cancer diagnosis and treatment [14].

There is a paucity of published data and limited understanding of the unmet needs of cancer patients in the Middle East, particularly in the Gulf Cooperation Council (GCC) countries, consisting of Kuwait, Qatar, Oman, Saudi Arabia, Bahrain and the United Arab Emirates (UAE). Identifying and addressing these unmet needs can lead to many positive patient outcomes, including better ability to cope with disease symptoms and treatment side-effects, improved physician-patient communication and better adherence to treatment regimens [7, 15]. The purpose of this study is to assess the unmet needs of cancer patients in the UAE, with the goal of improving the supportive care services for these patients and, thereby, positively impacting their overall quality of life [15].

\section{Methods}

A cross-sectional survey was conducted by a bilingual (Arabic and English) physician and, nurse researcher between December 2014 and December 2016 at a tertiary care, Joint Commission International accredited hospital and a regional oncology referral centre. The validated short form of the SCNS-SF34 was used for the study, primarily because of its feasibility and ease of use and coverage of many of the major domains of unmet needs [7]. The SCNS-SF34 assesses cancer-specific perceived needs across five analytically derived domains: psychological (10 items), health system information (11 items), patient care and support (5 items), physical and daily living (5 items) and sexuality (3 items) [16]. The SCNS-SF34 was provided by Dr. Allison Boyes (University of Newcastle upon Tyne, Australia) [16]. Prior to study commencement, the SCNS-SF34 was translated by a certified legal translator into Arabic, the preferred local language of the patients, and then, back translated into English to ensure the quality of the translation and to ensure that the structure, content and intent of the survey items did not alter during translation. Both the English $(n=14)$ and Arabic $(n=17)$ versions were pretested for reliability in the local setting using 31 patients from the oncology outpatient clinic.

Participating subjects were ambulatory patients diagnosed with cancer who presented to the oncology outpatient clinics. Potential participants were randomly selected using the patient appointment booking module of Malaffi, the hospital information system. Inclusion 
criteria included men or women aged 18-75 with a confirmed new or recurrent cancer diagnosis, who were informed of the cancer diagnosis and were capable of providing informed consent and completing the survey questionnaire, based on the European Cooperative Oncology Group (ECOG) status (0-3) [17]. Patient participants unable to meet the ECOG status, patients with mental or cognitive disorders and those unable to understand or unwilling to provide informed consent were excluded from the study. Respondents were asked to indicate their level of need for help during the last month for each item on the survey on a 5-point Likert scale, with the following response options: $1=$ no need, not applicable; $2=$ no need, satisfied; $3=$ low need; $4=$ moderate need and $5=$ high need [18]. Survey participants who selected a score of 4 or 5 indicated moderate or high unmet needs that required assistance [16]. Additional information, if required, was obtained from the patient's medical record and the hospital's cancer registry. Written consents were obtained from the respondents.

Data were analysed using SPSS Statistical Software Version 20 (SPSS Inc. Chicago, USA). The domains were identified as reported elsewhere [16]. The widely used principal component analysis (PCA) was used as the extraction method to undertake exploratory factor analysis; and varimax rotation was used to rotate the factors to better fit the data [19]. Convergent validity to assess if the survey items converged to measure a construct was also conducted using the correlation coefficient matrix method [19]. The percentage of total variance by each factor was calculated and pattern matrix was used to identify the domains. Kiaser-Meyer-Olkin (KMO) sampling adequacy and Bartlett's tests (to assess the strength of the relationship among the variables) were also applied to the construct [19]. The reliability of the inventory and its subscales were tabulated using Cronbach's alpha. In addition to descriptive statistics, the Chi-square test was adopted to assess the association between variables. Participant responses to each of the items were tabulated as mean \pm SEM. Comparison of means, when required, was accomplished using MedCalc software [20]. The study was approved by the regional research ethics committee (AAMDHREC 12/55).

\section{Results}

Approximately 300 patients with either a new cancer diagnosis or a diagnosed recurrence are treated at these oncology outpatient clinics every year. Based on our previous experience of a fully completed survey return rate of $80 \%$ in this population [19], 34 more participants were added to the original sample size of 169 (confidence level 95\%, confidence interval 5\%), obtaining a minimum sample size of 203 patient participants for the study. Two hundred and ten completed questionnaires were returned out of 268 administered $(78.3 \%$ response rate). The demographics of the patient participants are given in Table 1. The majority of the survey respondents were female $(69 \%$, $n=144)$, married $(76 \%, n=159)$, with college-level education $(54 \%, n=113)$.

Comparison of the five analytically derived domains indicated a significantly high psychological need $(3.63 \pm 0.023)$, followed by physical and daily living needs $(3.04 \pm 0.029, p<0.001)$, health system information needs $(3.03 \pm 0.02, p<0.001)$, patient care and support $(2.95$ $\pm 0.24, p<0.001$ ), with low sexuality needs $(1.79 \pm 0.08, p<0.001)$ (Figure 1). Five out of 10 items $(50 \%)$ from the psychological domain constituted the 10 most prevalent unmet moderate or high needs of oncology patients in the UAE, including concerns regarding an uncertain future ( $80 \%)$ and feelings about death and dying (77.1\%) (Table 2). Moderate or high feelings of lack of energy and tiredness $(75.2 \%)$, as well as pain affecting their physical and daily living activities $(74.8 \%)$, were prevalent in more than three-quarters of the participants. Needs related to sexuality, such as receiving information about sexual relationships (16.7\%) and changes in sexual feelings (9.5\%), scored low (no need or satisfied) for almost $85 \%$ of the patient population surveyed, data not shown.

Table 3 reports gender differences in participant responses. Gender correlated strongly with sexuality (changes in sexual feelings), with men expressing higher unmet need than women $(r=0.901, p<0.001)$. Women had significantly higher psychological unmet needs of 'feeling down or depressed' $(r=0.42, p<0.001)$ and 'feelings of sadness' $(r=0.26, p<0.001)$, as compared with male respondents. The need to be informed about cancer directly from the doctor, rather than a relative, was also moderately high among women $(r=0.37$, $p<0.001)$. Physical limitation, such as 'feeling unwell a lot of time', positively correlated $(r=0.28, p<0.001)$ with the unmet needs for women. 'Uncertainity about the future' (psychological) was directly correlated with patient age $(r=0.14, p<0.052$-tailed significance, data not shown).

Cultural differences in responses amongst the patient respondents are givenn in Table 4. UAE nationals reported higher unmet needs in the health system information domain. No other statistically significant differences in reports of unmet supportive care needs were noted between UAE nationals and non-nationals, or between Arabs and non-Arabs (data not shown). 


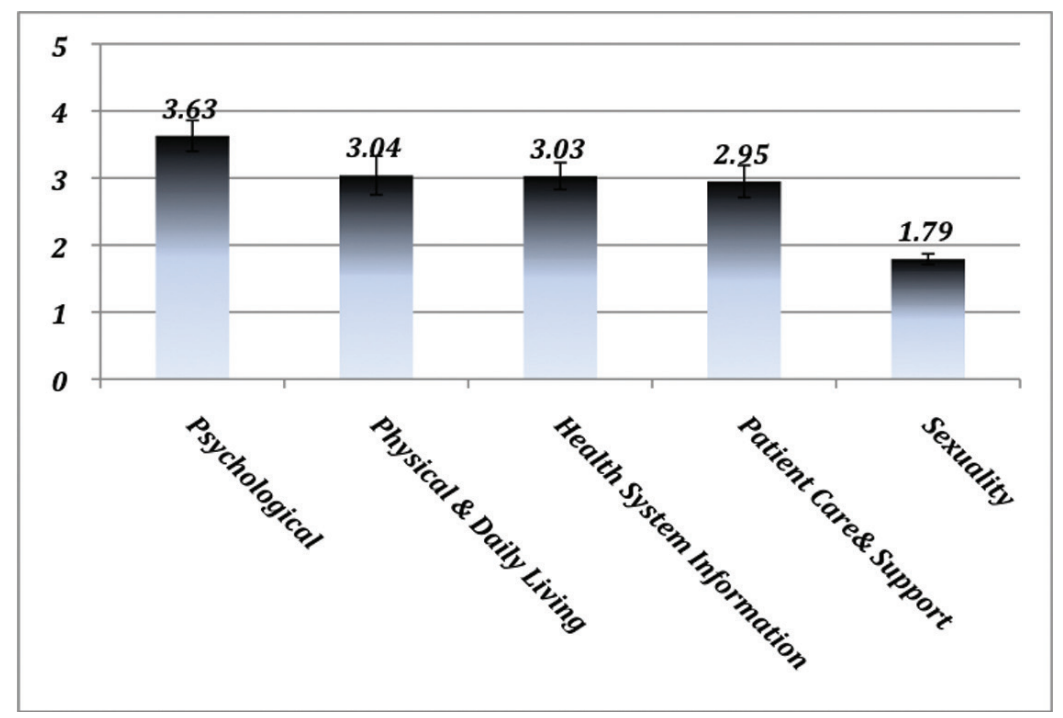

Figure 1. Patient responses to the SCNS-SF34-A survey are indicated as Mean \pm SEM for each of the five domains: psychological, physical and daily living, health system information, patient care and support and sexuality $(N=210)$.

Table 1. Demographics of the patient participants $(\mathrm{N}=\mathbf{2 1 0})$.

\begin{tabular}{|c|c|c|c|c|c|c|}
\hline Categories & $n$ & $\%$ & & Categories & $n$ & $\%$ \\
\hline Gender & & & & Education & & \\
\hline Female & 144 & 69 & & None & 37 & 18 \\
\hline Male & 66 & 31 & & > HighSchool & 113 & 54 \\
\hline Age & & & & Children (\#) & & \\
\hline $18-20 \mathrm{Y}$ & 2 & 1 & & None & 27 & 13 \\
\hline $21-30 \mathrm{Y}$ & 9 & 4 & & Less than 5 & 141 & 67 \\
\hline $31-50 \mathrm{Y}$ & 99 & 47 & & $5+$ & 42 & 20 \\
\hline $51-70 \mathrm{Y}$ & 79 & 38 & $N=210$ & Malignancy & & \\
\hline $71+Y$ & 18 & 9 & & Breast & 126 & 60 \\
\hline Nationality & & & & Gynaecological & 18 & 9 \\
\hline UAE/GCC & 45 & 21 & & Testicular & 17 & 8 \\
\hline Middle East & 95 & 45 & & Prostate & 19 & 9 \\
\hline Asia & 56 & 27 & & Lung & 21 & 10 \\
\hline Africa & 9 & 4 & & Others & 9 & 4 \\
\hline Marital status & & & & Cancer stage & & \\
\hline Single & 28 & 13 & & 1 & 55 & 26 \\
\hline Married & 159 & 76 & & II & 94 & 45 \\
\hline Others & 23 & 11 & & III/IV & 61 & 29 \\
\hline
\end{tabular}


Table 2. Top ten unmet supportive care needs of cancer patients.

\begin{tabular}{|c|c|c|c|c|}
\hline \multicolumn{5}{|c|}{ Moderate to high } \\
\hline Domain & What was your level of need for help with: & Mean response \pm SEM & $n$ & $\%$ \\
\hline Psychological & Uncertain about the future & $4.14 \pm 0.088$ & 168 & 80.0 \\
\hline Health system information & Being informed about your test results as soon as feasible & $4.0 \pm 0.052$ & 166 & 79.0 \\
\hline Psychological & Anxiety & $4.28 \pm 0.071$ & 165 & 78.6 \\
\hline Psychological & Feelings about death and dying & $4.14 \pm 0.090$ & 162 & 77.1 \\
\hline Physical and daily living & Lack of energy/tiredness & $3.98 \pm 0.082$ & 158 & 75.2 \\
\hline Physical and daily living & Pain & $4.17 \pm 0.063$ & 157 & 74.8 \\
\hline Psychological & Feelings of sadness & $4.08 \pm 0.079$ & 157 & 74.8 \\
\hline Health system information & $\begin{array}{l}\text { Receiving information about cancer test results to you } \\
\text { rather than to your relative or family }\end{array}$ & $4.08 \pm 0.072$ & 151 & 72 \\
\hline Health system information & $\begin{array}{l}\text { Being given explanations of those tests for which you would } \\
\text { like an explanation }\end{array}$ & $3.49 \pm 0.060$ & 135 & 64.3 \\
\hline Psychological & Keeping a positive outlook & $3.72 \pm 0.092$ & 129 & 61.4 \\
\hline
\end{tabular}

Table 3. Gender differences among cancer patients reporting unmet supportive care needs.

\begin{tabular}{|l|l|c|c|}
\hline \multicolumn{1}{|c|}{ Gender/domain } & What was your level of need for help with: & $\begin{array}{c}\text { Pearson correlation } \\
\text { coeffecient\{r\} }\end{array}$ & $\begin{array}{c}\text { Significance sig. } \\
\text { (2-tailed) }\end{array}$ \\
\hline Male/sexuality & Changes in sexual feeling & 0.901 & $p<0.001$ \\
\hline Female/psychological & Feeling down or depressed & 0.417 & $p<0.001$ \\
\hline Female/health system information & $\begin{array}{l}\text { Receiving information about cancer test results } \\
\text { to you rather than to your relative or family }\end{array}$ & 0.378 & $p<0.001$ \\
\hline Female/physical and daily living & Feeling unwell a lot of time & 0.281 & $p<0.001$ \\
\hline Female/psychological & Feelings of sadness & 0.261 & $p<0.001$ \\
\hline
\end{tabular}

Table 4. Comparison between UAE nationals and non-nationals (expatriates), cancer patients reporting unmet supportive care needs.

\begin{tabular}{|l|l|l|c|c|}
\hline \multicolumn{1}{|c|}{ Domain } & \multicolumn{1}{c|}{ Items } & \multicolumn{1}{c|}{ Population } & $\begin{array}{c}\text { Moderate/high need } \\
\boldsymbol{n}(\%)\end{array}$ & \multicolumn{1}{c|}{$\boldsymbol{P}$ Value } \\
\hline \multirow{2}{*}{ Health system information } & \multicolumn{1}{|c|}{$\begin{array}{l}\text { Being given explanations of those tests } \\
\text { for which you would like an explanation }\end{array}$} & UAE nationals $(n=47)$ & $36(76)$ \\
\cline { 2 - 5 } Health system information & $\begin{array}{l}\text { Being treated like a person not just } \\
\text { another case }\end{array}$ & UAE nationals $(n=47)$ & $95(61)$ \\
\cline { 2 - 5 } & Expatriates $(n=158)$ & $14(30)$ \\
\hline \multirow{2}{*}{0.001} & $25(16)$ \\
\hline
\end{tabular}

The consistency of the SCNS-SF-A was measured using the PCA, obtaining a KMO value of 0.88 , reaching statistical significance. Bartlett's test of sphericity was significant (0.000). The PCA revealed the presence of a component with an eigen value of 14.9 , explaining $81.2 \%$ of total variance. Correlation coefficient indicating relationship between the items is given in Table 5. Cronbach's $\alpha$ reliability assessment of each of the four domains ranged between 0.73 and 0.84 (Table 5). The overall Cronbach's a reliability score for all 34 items for the final construct was high at 0.79 . 
Table 5. Reliability and validity of the SCNS34-A construct. Internal consistency of the inventory and its subscales were tabulated using Cronbach's alpha and PCA was used as the extraction method to undertake factor analysis.

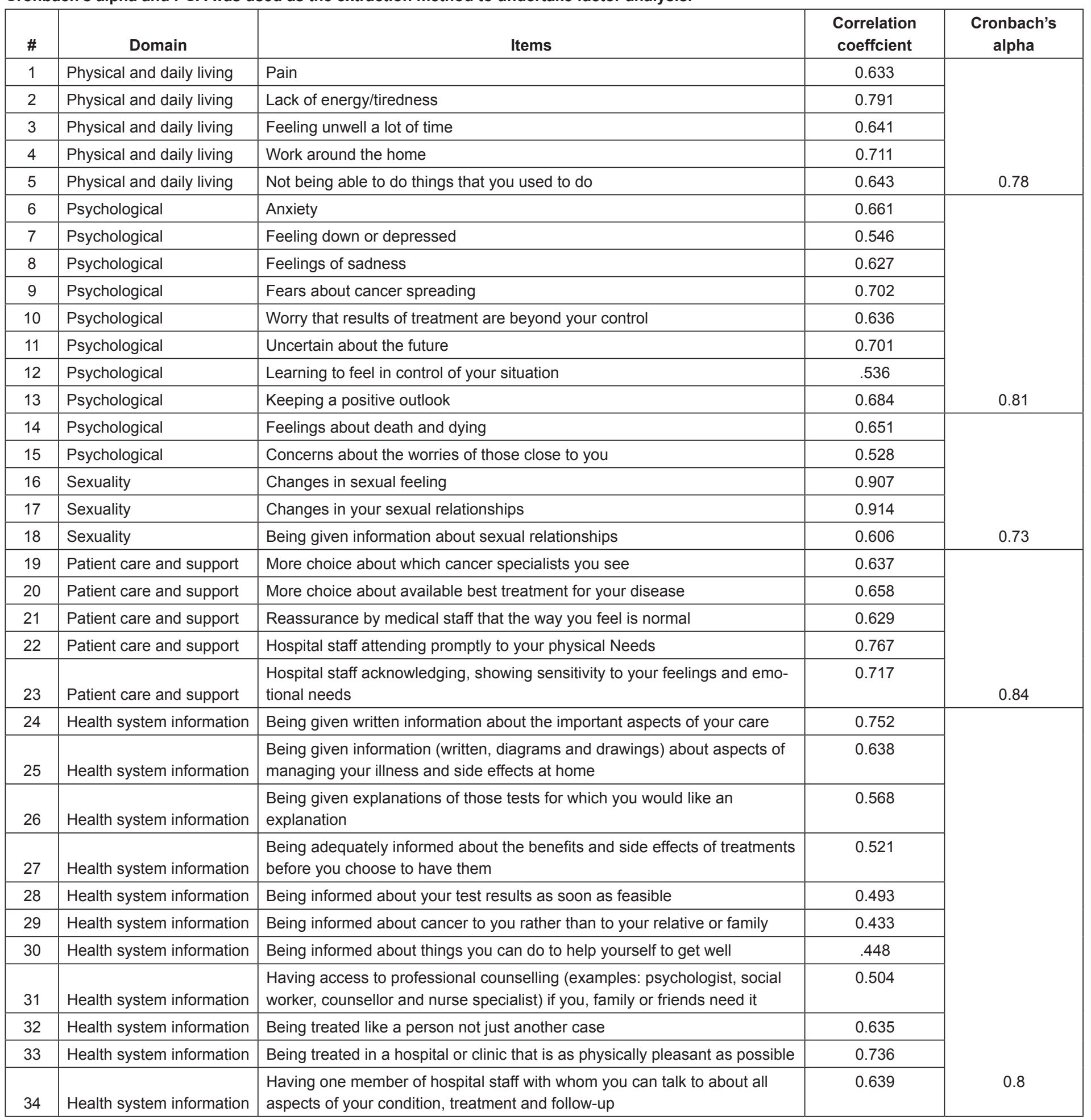




\section{Discussion}

Wellbeing in cancer patients can be considered a balance between two sets of factors: the stress and burden resulting from the cancer experience and the resources available for coping and mitigating stress and burden [10, 11]. Our study demonstrates that cancer patients in the UAE experience a wide range of unmet supportive needs, primarily related to psychological needs. Amongst the critical top 10 unmet needs of the patients surveyed, $50 \%$ were psychological in nature, with women expressing significantly higher psychological needs than men. This finding suggests that the improvement of mental health services for cancer patients is an urgent priority. Proactive encouragement and recruitment of female cancer patients into counselling services can help mitigate some of their needs.

In addition, the high prevalence of physical symptoms, including lack of energy, tiredness and pain limiting physical and daily living, is quite concerning. This highlights the need to focus on early and adequate pain management and physical rehabilitation as important components of cancer care. Oncology units in the UAE should consider inculcating these specialities into multidisciplinary cancer care teams. It has also been reported that patients have several misconceptions regarding pain, specifically that increasing pain signifies disease progression, medicine to control pain may weaken the immune system and pain is inevitable for cancer patients [21]. These beliefs identify a need for cancer support groups to address misinformation and help manage patient needs. A large body of literature demonstrates that participation in cancer support groups improves patients' quality of life and wellbeing [22, 23]. As such, the development of this service at oncology centers throughout the UAE can serve as viable channels of information and help to meet several of the psychological needs of cancer patients.

Furthermore, the unmet need of cancer patients to be informed about their diagnostic tests in a timely manner and to be provided an explanation about their test results indicate that system changes are required. In the UAE, it is common practice to send diagnostic specimens overseas for genetic and mutational analysis, leading to delays in reporting results. The inclusion of genetic counsellors as part of the multidisciplinary care teams for cancer management can provide accurate, timely information and help alleviate some patient fears during the diagnostic process. The finding that women were more concerned about receiving test results directly from the doctor, instead of a family member, is not surprising given that the UAE, as much of the Arab world, is a patriarchal society where physicians often receive consent from, and discuss results with, male family members instead of female patients. It is important that cancer care health professionals understand the need for all patients to be included in treatment discussions. Actively involving women in all treatment decisions will help to alleviate this concern. Also, the addition of social workers into cancer care teams to directly address female patient needs may help resolve potential family dynamic issues.

Interestingly, sexuality scored low as an unmet need for cancer patients in our study, although men expressed higher unmet need for sexuality as compared to women $(r=0.901, p<0.001)$. This is consistent with previous studies demonstrating that patterns of unmet supportive care needs differ across cultures and health care services among Caucasians, Japanese and Chinese [24, 25, 26]. Previous studies, based on Hong Kong Chinese women with breast cancer, also showed similar low unmet needs related to sexuality [12, 13, 18, 21, 26]. In the $\mathrm{UAE}$, it is not standard practice to routinely assess sexual wellbeing in cancer and palliative care patients, as it is often not a presenting symptom. Also, it is possible that the reported low sexual needs were due to an unwillingness of the patient to discuss sexual concerns. Routinely addressing sexual issues as part of cancer treatment may overcome some of these cultural barriers and ensure that this aspect of patient care needs is met.

The study did not reveal significant cultural differences in unmet supportive care needs among the oncology patients in the UAE, perhaps because most respondents were from in and around the Arab world. As all patients were residents of the UAE, it is also possible that respondents had already assimilated to UAE culture.

The strengths of this study include the high response rate and participation of multi-ethnic patients. We also attempted to minimise the effect of social desirability response bias by assuring respondent confidentiality. Our results, however, should be viewed in light of some limitations. First, the cross-sectional study design provides correlation, but not causal inference. Patients from only two institutions were surveyed; however, both participating hospitals have large oncology units and one is the regional referral centre for the UAE and neighbouring countries. Also, most patient participants had breast or gynaecologic malignancies. Perspectives of patients with other malignancies, including gastrointestinal cancers, are important. Finally, inherent to any self-report of complex issues, such as quality of life, there are influencing factors that may not have been fully addressed in this study, including family/caregiver and social support, spirituality and patient individual personality. Notwithstanding these limitations, to our knowledge, this is the first study in the region that has assessed the 
supportive care needs of cancer patients and provides valuable information to improve patient care. Identifying and understanding cancer patient perspectives is a first step in the development of hospital and community services to meet their needs. Future studies focusing on the implementation of integrated multidimensional approaches to cancer support services are needed to help improve the quality of UAE cancer patients' and families' lives.

\section{Conclusion}

As the cancer rates climb in the UAE, it is important to fully understand and meet the needs of oncology patients. Our study has identified several opportunities to improve the care and support services available. Oncology centres in the UAE should develop an integrated, multifaceted approach to identify and meet the supportive care needs of cancer patients. The improvement of mental health services for oncology patients, development of multidisciplinary cancer care teams, introduction of cancer support groups and fully engaging women in all treatment discussions and decisions, are all feasible and easy to implement interventions that can significantly improve patient care and wellbeing. As the UAE is a multi-ethnic and multicultural society, the findings of this study may prove to be useful in addressing the needs of cancer patients in the pan Arab region.

\section{Acknowledgments}

Ghada Houssneh, Charge Nurse, Breast Care Center, Tawam Hospital is acknowledged for her technical help with data collection. Professor Allison Boyes, Newcastle, Australia is thanked for the SCNS-SF survey and scoring system provided to us in order to complete the study.

\section{Conflicts of interest}

The authors declare no conflicts of interest.

\section{Funding declaration}

The study was not funded by any funding sources.

\section{References}

1. Lozano R, Naghavi M, and Foreman K, et al (2012) Global and regional mortality from 235 causes of death for 20 age groups in 1990 and 2010: a systematic analysis for the Global Burden of Disease Study 2010 Lancet 380(9859) 2095-2128 https://doi. org/10.1016/S0140-6736(12)61728-0 PMID: 23245604

2. Allemani C, Weir HK, and Carreira H, et al (2015) Global surveillance of cancer survival 1995-2009: analysis of individual data for 25,676,887 patients from 279 population-based registries in 67 countries (CONCORD-2) Lancet 385(9972) 977-1010 https:// doi.org/10.1016/S0140-6736(14)62038-9 PMCID: 4588097

3. Charafeddine MA, Olson SH, and Mukherji D, et al (2017) Proportion of cancer in a Middle Eastern country attributable to established risk factors BMC Cancer 17(1) 337 https://doi.org/10.1186/s12885-017-3304-7 PMID: 28521815 PMCID: $\underline{5437575}$

4. World Health Organization, Regional Office for the Eastern Mediterranean (2010) Strategy for cancer prevention and control in the Eastern Mediterranean Region 2009-2013 Geneva: WHO [http://applications.emro.who.int/dsaf/EMRPUB 2010_1278.pdf] Date accessed: $23 / 04 / 17$ 
5. Miller KD, Siegel RL, and Lin CC, et al (2016) Cancer treatment and survivorship statistics, 2016 CA Cancer J Clin 66(4) 271-289 https://doi.org/10.3322/caac.21349 PMID: 27253694

6. Teshome M and Hunt KK (2014) Neoadjuvant therapy in the treatment of breast cancer Surg Oncol Clin N Am 23(3) 505-523

7. Okediji PT, Salako O, and Fatiregun $\mathrm{OO}$ (2017) Pattern and predictors of unmet supportive care needs in cancer patients Cureus 9(5) e1234 PMID: 28620565 PMCID: $\underline{5467772}$

8. Gregurek R, Bras M, and Dordević V, et al (2010) Psychological problems of patients with cancer Psychiatr Danub 22(2) 227-230

9. Paraskevi T (2012) Quality of life outcomes in patients with breast cancer Onc Rev 6(1) https://doi.org/10.4081/oncol.2012.e2 PMID: 25992204 PMCID: $\underline{4119638}$

10. Wagner EH, Aiello Bowles EJ, and Greene SM, et al (2010) The quality of cancer patient experience: perspectives of patients, family members, providers and experts Qual Saf Health Care 19(6) 484-489

11. Harrison JD, Young JM, and Price MA, et al (2009) What are the unmet supportive care needs of people with cancer? A systematic review Support Care Cancer 17(8) 1117-1128 https://doi.org/10.1007/s00520-009-0615-5 PMID: 19319577

12. Lam WW, Au AH, and Wong JH, et al (2011) Unmet supportive care needs: a cross-cultural comparison between Hong Kong Chinese and German Caucasian women with breast cancer Breast Cancer Res Treat 130(2) 531-541 https://doi.org/10.1007/ s10549-011-1592-1 PMID: 21617919

13. Coreil J, Wilke J, and Pintado I (2004) Cultural models of illness and recovery in breast cancer support groups Qual Health Res 14 905-923 https://doi.org/10.1177/1049732304266656 PMID: 15296663

14. Daher M (2012) Cultural beliefs and values in cancer patients Ann Oncol $366-69$ https://doi.org/10.1093/annonc/mds091

15. Brédart A, Kop JL, and Griesser AC, et al (2012) Validation of the 34-item supportive care needs survey and 8-item breast module French versions (SCNS-SF34-Fr and SCNS-BR8-Fr) in breast cancer patients Eur J Cancer Care 21(4) 450-459 https:// doi.org/10.1111/j.1365-2354.2012.01356.x

16. Boyes A, Girgis A, and Lecathelinais C (2009) Brief assessment of adult cancer patients' perceived needs: development and validation of the 34-item supportive care needs survey (SCNS-SF34) J Eval Clin Pract 15(4) 602-606

17. Oken MM, Creech RH, and Tormey DC, et al (1982) Toxicity and response criteria of the Eastern Cooperative Oncology Group Am J Clin Oncol 5 649-655 https://doi.org/10.1097/00000421-198212000-00014

18. McElduff P, Boyes A, and Zucca A, et al (2004) The Supportive Care Needs Survey: A Guide to Administration, Scoring and Analysis (Newcastle: Centre for Health Research and Psycho-Oncology) pp 1-69

19. Nair SC, Satish KP, and Sreedharan J, et al (2016) Assessing health literacy in the eastern and middle-eastern cultures $B M C$ Public Health 16831 https://doi.org/10.1186/s12889-016-3488-9 PMID: 27538529 PMCID: $\underline{4991078}$

20. [https://www.medcalc.org/calc/comparison_of means.php] Date accessed: 02/03/17

21. Nayak MG, George A, and Vidyasagar M, et al (2015) Symptoms experienced by cancer patients and barriers to symptom management Indian J Pall Care 21(3) 349-354 https://doi.org/10.4103/0973-1075.164893

22. Lindemalm C, Strang P, and Lekander M (2005) Support group for cancer patients. Does it improve their physical and psychological wellbeing? A pilot study Support Care Cancer 13(8) 652-657 https://doi.org/10.1007/s00520-005-0785-8 PMID: 16041464

23. Ussher J, Kirsten L, and Butow P, et al (2006) What do cancer support groups provide which other supportive relationships do not? The experience of peer support groups for people with cancer Soc Sci Med 62(10) 2565-2576 https://doi.org/10.1016/j. socscimed.2005.10.034 
24. Li WW, Lam WW, and Shun SC, et al (2013) Psychometric assessment of the Chinese version of the Supportive Care Needs Survey short-form (SCNS-SF34-C) among Hong Kong and Taiwanese Chinese colorectal cancer patients PLoS One 8(10) e75755 https://doi.org/10.1371/journal.pone.0075755 PMID: 24146774 PMCID: 3795709

25. Fielding R, Lam WWT, and Shun SC, et al (2013) Attributing variance in supportive care needs during cancer: culture-service, and individual differences, before clinical factors PLoS One 8(5) e65099 https://doi.org/10.1371/journal.pone.0065099 PMID: 23741467 PMCID: $\underline{3669056}$

26. Wen KY, Fang CY, and Ma GX (2014) Breast cancer experience and survivorship among Asian Americans: a systematic review J Cancer Surviv 8(1) 94-107 https://doi.org/10.1007/s11764-013-0320-8 PMCID: $\underline{3945715}$ 